\title{
SIMPLE KNOTS WHICH ARE DOUBLY-NULL-COBORDANT
}

\section{KEARTON}

ABSTRACT. Using the Seifert matrix, a necessary and sufficient condition is given for a simple $(2 q-1)$-knot, $q>1$, to be doubly-nullcobordant.

An $n$-knot is a smooth oriented submanifold $K$ of the $(n+2)$-sphere $S^{n+2}$, where $K$ is homeomorphic to $S^{n}$. An $n$-knot is doubly-null-cobordant if it is a cross-section of an unknotted pair $\left(S^{n+3}, S^{n+1}\right)$. D. W. Sumners [3] has studied the case $n=2 q-1$, and has given a necessary condition for $K$ to be doubly-null-cobordant. He has also given a partial converse, which it is the purpose of this paper to strengthen.

Let $K$ be a $(2 q-1)$-knot; $K$ bounds a smooth oriented submanifold $U$ of $S^{2 q+1}$, and choosing a basis for the torsion-free part of $H_{q}(U)$, we obtain a matrix $A$ of linking numbers called a Seifert matrix of $K$. The submanifold $U$ is not unique, but any two such submanifolds are cobordant, and this induces an equivalence relation on the Seifert matrices of $K$ which may be described as follows. An elementary enlargement of $A$ is an integer matrix of the form

$$
\left(\begin{array}{c|cc}
A & 0 & 0 \\
\hline \alpha & 0 & 0 \\
0 & 1 & 0
\end{array}\right) \text { or }\left(\begin{array}{c|cc}
A & \beta & 0 \\
\hline 0 & 0 & 1 \\
0 & 0 & 0
\end{array}\right)
$$

where $\alpha$ is a row vector, $\beta$ a column vector. We say $A \sim B$ if $A$ is an elementary enlargement of $B$ or vice versa, or if there is a unimodular congruence between $A$ and $B$. Two Seifert matrices $A, B$ are equivalent if they are connected by a sequence $A=A_{1}, \ldots, A_{k}=B$ such that $A_{i} \sim A_{i+1}$ for $1 \leq i<k$.

An elementary enlargement is trivial if $\alpha=0$ or $\beta=0$, and a succession of trivial elementary enlargements is a trivial enlargement.

The Seifert matrix $A$ is doubly-null-cobordant if it is congruent to one of the form $\left(\frac{0}{*} * 0\right)$, all the blocks being square.

Levine [2] defines a simple $(2 q-1)$-knot as one whose complement has the homotopy $(q-1)$-type of a circle. If $K$ is a $(2 q-1)$-knot which bounds a $(q-1)$-connected submanifold $U$, then the associated matrix $A$ is said to be special; we remark that this can occur precisely when $K$ is simple.

Received by the editors July 12, 1974 . 57D40.

AMS (MOS) subject classifications (1970). Primary 57C45, 55A25; Secondary

Key words and phrases. Simple knot, doubly-null-cobordant, Seifert matrix. 
Lemma. Let $A$ be a Seifert matrix of a simple $(2 q-1) \circ k n o t K$. If $q>$ 2 , then $A$ is special; if $q=2$, then some trivial enlargement of $A$ is special.

Proof. Let $U$ be a submanifold with which $A$ is associated. By results of [1], $U$ is cobordant to a $(q-1)$-connected submanifold $V$, and so there exists a sequence $A=A_{1}, \ldots, A_{k}=B$ where $A_{i} \sim A_{i+1}$ for $1 \leq i<k$, and $B$ is a Seifert matrix associated with $V$. If $q>2$, it is shown in $[2, \$ \$ 11-$ 13] that if $C \sim D$ and $D$ is a special Seifert matrix, then so is $C$. But $B$ is special, hence so is $A$. If $q=2$, the arguments in [2, $\$ 10-13]$ apply provided we replace the sequence $A_{1}, \ldots, A_{k}$ by $A_{1}^{\prime}, \ldots, A_{k}^{\prime}$, each $A_{i}^{\prime}$ being a trivial enlargement of $A_{i}$ with order $A_{i}^{\prime}-\operatorname{order} A_{i}$ independent of $i$.

Theorem. If $K$ is a simple $(2 q-1)$-knot, $q \geq 2$, then $K$ is doubly-nullcobordant if and only if it possesses a doubly-null-cobordant Seifert matrix.

Proofo If $K$ is doubly-null-cobordant, then Sumners proves in [3] that it has a doubly-null-cobordant matrix. Assume conversely that $A$ is such a matrix; then any trivial enlargement of $A$ is also doubly-null-cobordant, and so by the Lemma we may take $A$ to be special. Another result of Sumners [3, Theorem 3.1] shows that $K$ is doubly-null-cobordant.

\section{REFERENCES}

1. Jerome Levine, Unknotting spheres in codimension two, Topology 4 (1965), 9-16. MR $31 \# 4045$.

2. - An algebraic classification of some knots of codimension two, Comment. Math. Helv. 45 (1970), 185-198. MR 42 \#1133.

3. D. W. Sumners, Invertible knot cobordisms, Comment. Math. Helv. 46 (1971), 240-256. MR $44 \# 7535$. TAIN

CORPUS CHRISTI COLLEGE, CAMBRIDGE UNIVERSITY, CAMBRIDGE, GREAT BRI- 